\title{
Reasons for Discontinuing Active Surveillance : Assessment of 21 Centres in 12 Countries in the Movember GAP3 Consortium
}

\section{Van Hemelrijck, Mieke}

2019-03

Van Hemelrijck, M , Ji , X, Helleman , J , Roobol , M J , van der Linden , W , Nieboer , D , Bangma, C H , Frydenberg, M , Rannikko , A, Lee , L S, Gnanapragasam , V J , Kattan , M W , Trock , B , Ehdaie , B , Carroll , P , Filson , C , Kim , J , Logothetis , C , Morgan , T , Klotz , L , Pickles , T , Hyndman , E , Moore , C M , Gnanapragasam , V , Van Hemelrijck , M , Dasgupta , P , Bangma , C , Roobol , M , Villers , A , Valdagni , R , Perry , A , Hugosson , J , Rubio-Briones , J , Bjartell , A, Hefermehl , L, Shiong , L L , Frydenberg , M , Kakehi , Y, Byung Ha Chung, van der Kwast , T , Obbink, H , Hulsen , T , de Jonge , C , Kattan , M , Xinge , J , Muir , K, Lophatananon , A , Fahey , M \& Steyerberg , E 2019 , ' Reasons for Discontinuing Active Surveillance : Assessment of 21 Centres in 12 Countries in the Movember GAP3 Consortium ' , European Urology , vol. 75 , no. 3 , pp. 523-531 . https://doi.org/10.1016/j.eururo.20

http://hdl.handle.net/10138/312565

https://doi.org/10.1016/j.eururo.2018.10.025

unspecified

publishedVersion

Downloaded from Helda, University of Helsinki institutional repository.

This is an electronic reprint of the original article.

This reprint may differ from the original in pagination and typographic detail.

Please cite the original version. 


\title{
Reasons for Discontinuing Active Surveillance: Assessment of 21 Centres in 12 Countries in the Movember GAP3 Consortium
}

\author{
Mieke Van Hemelrijck ${ }^{a, *}, X \mathrm{Ji}^{b}$, Jozien Helleman ${ }^{c}$, Monique J. Roobol ${ }^{c}$, Wim van der Linden ${ }^{d}$, \\ Daan Nieboer ${ }^{c}$, Chris H. Bangma ${ }^{c}$, Mark Frydenberg ${ }^{e, f}$, Antti Rannikko $^{g}$, Lui S. Lee ${ }^{h}$, \\ Vincent J. Gnanapragasam ${ }^{i}$, Mike W. Kattan ${ }^{b}$, Members of the Movember Foundation's Global \\ Action Plan Prostate Cancer Active Surveillance (GAP3) consortium ${ }^{\dagger}$
}

${ }^{\mathrm{a}}$ Translational Oncology Er Urology Research (TOUR), School of Cancer and Pharmaceutical Sciences, King's College London, London, UK; ' ${ }^{\mathrm{b}}$ Department of Quantitative Health Sciences, Cleveland Clinic, Cleveland, OH, USA; ${ }^{\mathrm{c}}$ Department of Urology, Erasmus University Medical Center, Rotterdam, The Netherlands; ${ }^{\mathrm{d}}$ Department of Professional Health Solutions \& Services, Philips Research, Eindhoven, The Netherlands; ${ }^{\mathrm{e}}$ Department of Surgery, Monash University, Melbourne, Australia; ${ }^{\mathrm{f}}$ Department of Urology, Monash Health, Melbourne, Australia; ${ }^{\mathrm{g}}$ Helsinki University and Helsinki University Hospital, Helsinki, Finland; ${ }^{\mathrm{h}}$ Department of Urology, Singapore General Hospital, Singapore; ${ }^{\mathrm{i}}$ Academic Urology Group, Department of Surgery and Oncology, University of Cambridge, Cambridge, UK

Article info

Article history:

Accepted October 13, 2018

Associate Editor:

James Catto

Keywords:

Prostate cancer

Active surveillance

Discontinuation

Worldwide

\begin{abstract}
Background: Careful assessment of the reasons for discontinuation of active surveillance (AS) is required for men with prostate cancer (PCa).

Objective: Using Movember's Global Action Plan Prostate Cancer Active Surveillance initiative (GAP3) database, we report on reasons for AS discontinuation.

Design, setting, and participants: We compared data from 10296 men on AS from 21 centres across 12 countries.

Outcome measurements and statistical analysis: Cumulative incidence methods were used to estimate the cumulative incidence rates of AS discontinuation.

Results and limitations: During 5-yr follow-up, 27.5\% (95\% confidence interval [CI]: 26.4$28.6 \%$ ) men showed signs of disease progression, $12.8 \%$ (95\% CI: $12.0-13.6 \%$ ) converted to active treatment without evidence of progression, $1.7 \%$ (95\% CI: $1.5-2.0 \%)$ continued to watchful waiting, and $1.7 \%$ (95\% CI: 1.4-2.1\%) died from other causes. Of the 7049 men who remained on AS, 2339 had follow-up for $>5 \mathrm{yr}, 4561$ had follow-up for $<5 \mathrm{yr}$, and 149 were lost to follow-up. Cumulative incidence of progression was $27.5 \%$ (95\% CI: $26.4-28.6 \%)$ at $5 \mathrm{yr}$ and $38.2 \%$ (95\% CI: $36.7-39.9 \%$ ) at $10 \mathrm{yr}$. A limitation is that not all centres were included due to limited information on the reason for discontinuation and limited follow-up.

Conclusions: Our descriptive analyses of current AS practices worldwide showed that $43.6 \%$ of men drop out of AS during 5-yr follow-up, mainly due to signs of disease progression. Improvements in selection tools for AS are thus needed to correctly allocate men with PCa to AS, which will also reduce discontinuation due to conversion to active treatment without evidence of disease progression.

Patient summary: Our assessment of a worldwide database of men with prostate cancer (PCa) on active surveillance (AS) shows that $43.6 \%$ drop out of AS within $5 \mathrm{yr}$, mainly due to signs of disease progression. Better tools are needed to select and monitor men with PCa as part of AS.

(C) 2018 European Association of Urology. Published by Elsevier B.V. All rights reserved.

${ }^{\dagger}$ The Movember Foundation's Global Action Plan Prostate Cancer Active Surveillance (GAP3) consortium members are presented in the Supplementary material.

* Corresponding author. TOUR-King's College London, Guy's Hospital, 3rd floor Bermondsey Wing, London SE1 9RT, UK. Tel. +44 2071885594; Fax: +44 2071889986.

E-mail address: Mieke.vanhemelrijck@kcl.ac.uk (M. Van Hemelrijck).
\end{abstract}




\section{Introduction}

About 2 decades ago, the concept of active surveillance (AS) was introduced as a management strategy for men with lowrisk prostate cancer ( $\mathrm{PCa}$ ) [1]. Men are monitored closely through repeated prostate-specific antigen (PSA) measurements, biopsies, and potentially also magnetic resonance imaging (MRI), with the intention to start curative treatment when their PCa is reclassified as higher risk due to signs of progression (ie, clinical or pathological) and to minimise the harm caused by the overtreatment of indolent cancer [2]. However, even though AS has no long-term physical morbidity, studies continue to report that $1.6-38 \%$ of men opt out of AS, often with no or little evidence of disease progression, within $5 \mathrm{yr}$ [3]. Thus, men embarking on AS are likely to transition to an alternative strategy within a decade, which highlights the need for more insight into AS protocols [1,4].

Careful assessment of reasons for discontinuation of AS is required, especially since treatment pathways for men with low-risk PCa vary by country and are managed differently in various healthcare systems $[5,6]$. It is unclear whether a decrease in health-related quality of life in men on AS precipitates their transition to radical treatment or whether this is driven by the distress over disease progression, physiological symptoms, or the burden of age. Most studies are small and with short follow-up [7-10]. Better understanding of reasons for opting out of AS is thus needed to help define a management strategy for AS.

Hence, in 2014, the Movember Foundation launched the Global Action Plan Prostate Cancer Active Surveillance initiative (GAP3), which covers the largest centralised PCa AS database to date (https://gap3.movemberprojects.com). Its primary goal is to create a global consensus with uniform guidelines on the selection and monitoring of men with low-risk PCa [11]. Here, we report on adherence to AS and the reasons for discontinuation by comparing data from 10296 men on AS from 21 different centres across 12 different countries.

\section{Patients and methods}

\subsection{Study population}

Between 2014 and 2016, the global GAP3 database was created by combining patient data from established AS cohorts worldwide. Requirements for participation included, amongst others, ethical approval for sharing digital patient data in a centralised global database and active registry of AS patients over the last $2 \mathrm{yr}$ or more, including at least $\sim 50$ patients annually. To date, 25 centres from the USA, Canada, Australasia, the UK, and Europe fulfilled the requirements for participation and joined the initiative [11], resulting in data for a total of 15101 men on AS. For the current study, we excluded 3084 patients from Dublin, MD Anderson Cancer Centre, Toronto, and University of Michigan and Michigan Urological Surgery Improvement Collaborative, as these centres did not distinguish between progression and anxiety events. Furthermore, to ensure as much homogeneity in our AS cohort as possible, we included only men with a Gleason grade group of 1 , leaving 10296 patients for the final analysis. Each institution obtained institution ethical approval and signed a Movember end user license agreement, an access rights principles agreement, and the commonly agreed upon GAP3 analytical plan.
Although there are many variations in existing protocols, most agreed that the most suitable patients for AS are those with age $>18 \mathrm{yr}$, pretreatment clinical stage T1-T2, serum PSA $\leq 10 \mathrm{ng} / \mathrm{ml}$, a biopsy Gleason grade group of 1 or 2, and a maximum of two tumour-positive biopsy core samples. The AS inclusion criteria for the 25 centres are shown in Supplementary Table 1 [11]. Some protocols included PSA density (most often using a cut-off of $0.2 \mathrm{ng} / \mathrm{ml}$ ), the maximum extent of cancer per core (most often using a cut-off of 50\%), life expectancy of $>10 \mathrm{yr}$, and adequate biopsy sampling as inclusion criteria for AS. An overview of contemporary worldwide AS practices across the world (and included in GAP3) can also be found in the systematic review by Kinsella et al. [3] and the cohort profile of the GAP3 database [5].

Following initiation of AS, almost all protocols recommended serial measurements (with a variation in time intervals) of serum PSA levels, digital rectal examination, and surveillance biopsy sampling in order to identify pathological progression. Several protocols considered MRI for routine use in AS, again with many differences between recommended frequencies. An overview of the AS follow-up protocols of the 25 institutes included in GAP3 is given in Supplementary Table 2 [11].

In addition to baseline criteria for selection and monitoring of AS, the GAP3 database also contains information on discontinuation of AS (ie, the reasons for stopping AS), and potential following treatments (eg, radical prostatectomy) and cause of death. Each centre reports for each patient an event time, defined as the time from the patient's AS initiation to discontinuation of AS due to "conversion to watchful waiting", "clinical progression", "pathological progression", "clinical and pathological progression", "PSA progression (PSA doubling time <3 yr)", "other PSA kinetics", "patient choice/anxiety", "doctor's anxiety", "radiological progression", "death", “loss to follow-up", “other/unknown reasons", or "still being on AS". These events are defined according to the centres' own criteria. We used the following coding for defining the signs of disease progression: "clinical and pathological progression", "clinical progression", "other PSA kinetics", "pathological progression", "PSA progression", and "radiological progression". If the reason for discontinuation was classified as "other/ unknown", but the "pathological progression status" reported at the time of AS discontinuation was "Gleason grade group 3 or higher" or the "clinical progression status" was "CT3 or higher" or "PSA progression status" was "PSA $>20$ ", the reason for discontinuation was also classified as signs of disease progression. The term "sign of disease progression" as used in this manuscript can thus refer to risk reclassification or disease progression as such. Conversion to active treatment without evidence of disease progression includes those patients for whom there was no information on specific discontinuation or disease progression (according to the criteria described above) and for whom specific treatment information was available, as well as those for whom the reason for discontinuation was registered as "doctor's anxiety" or "patient's choice/anxiety". The distribution of different types of active treatment has been described in detail in our recently published cohort profile [11].

\subsection{Statistical methods}

Descriptive statistics were used to summarise patient characteristics. The cumulative incidence method was used to estimate the rates of each event for discontinuation of AS. Cox proportional hazards regression analyses were used to estimate hazard ratios for various reasons of discontinuation based on age, PSA, and the number of positive biopsy cores. To account for the heterogeneity between centres, these models used the centre as a stratum. $\mathrm{R}$ version 3.3.2 (The $\mathrm{R}$ Foundation for Statistical Computing, Vienna, Austria) was used to perform all analyses.

\section{Results}

Table 1 shows the distribution of men on AS included in this study according to patient and tumour characteristics, by 
Table 1 - Distribution of men on AS according to patient and tumour characteristics, by outcome following AS at 5 yr of follow-up

\begin{tabular}{|c|c|c|c|c|c|c|}
\hline Variable & $\begin{array}{c}\text { Censor or still } \\
\text { on AS } \\
(N=7049)\end{array}$ & $\begin{array}{c}\text { Progression } \\
\text { treatment } \\
(N=2061)\end{array}$ & $\begin{array}{l}\text { Conversion to } \\
\text { active treatment } \\
\text { without evidence } \\
\text { of progression } \\
(N=952)\end{array}$ & $\begin{array}{l}\text { Watchful waiting } \\
\qquad(N=118)\end{array}$ & $\begin{array}{c}\text { Other cause } \\
\text { of death } \\
(N=116)\end{array}$ & $p$ value \\
\hline Years on AS & $3.3(1.4-5.8)$ & $1.4(1.1-2.5)$ & $1.6(1.0-2.7)$ & $1.7(1.2-3.1)$ & $2.3(1.5-3.4)$ & $<0.01$ \\
\hline Year of diagnosis & $2010(2007-2013)$ & $2010(2007-2011)$ & $2008(2004-2010)$ & 2010 (2009-2010) & $2007(2003-2010)$ & $<0.01$ \\
\hline Age at start of AS (yr) & $65(60-69)$ & $65(61-69)$ & $65(60-69)$ & $72(65-75)$ & $69(65-73)$ & $<0.01$ \\
\hline PSA at start of AS $(\mathrm{ng} / \mathrm{ml})$ & $5.3(3.9-7.2)$ & $5.4(4.2-7.0)$ & $5.6(4.2-7.3)$ & $5.9(4.5-7.5)$ & $6.4(4.2-9.1)$ & 0.01 \\
\hline Number of biopsy cores with PCa & $1(1-2)$ & $1(1-2)$ & $1(1-2)$ & $1(1-2)$ & $1(1-2)$ & $<0.01$ \\
\hline
\end{tabular}

outcome at $5 \mathrm{yr}$ of follow-up. During 5-yr follow-up, $27.5 \%$ (95\% confidence interval [CI]: 26.4-28.6\%) men showed signs of disease progression, $12.8 \%$ (95\% CI: $12.0-13.6 \%$ ) converted to active treatment without evidence of progression, $1.7 \%$ (95\% CI: $1.5-2.0 \%$ ) continued to watchful waiting, and $1.7 \%$ (95\% CI:1.4-2.1\%) died from other causes. Of the 7049 men who remained on AS during follow-up, 2339 had follow-up of $>5 \mathrm{yr}, 4561$ had $<5 \mathrm{yr}$ of follow-up, and 149 were lost to follow-up. Hence, at $5 \mathrm{yr}$ of follow-up, the cumulative incidence rate of men remaining on AS was $56.4 \%(95 \% \mathrm{CI}$ : 55.2-57.6\%), and 43.6\% (95\% CI: 42.4-44.8\%) were lost to follow-up or discontinued AS. Furthermore, the distribution of outcomes and tumour characteristics per participating centre are shown in Table 2 and Supplementary Table 3.
The cumulative incidence of signs of disease progression was $27.5 \%(95 \% \mathrm{CI}: 26.4-28.6 \%)$ at $5 \mathrm{yr}$ and $38.2 \%(95 \% \mathrm{CI}$ : $36.7-39.9 \%)$ at $10 \mathrm{yr}$. Figure 1 shows the cumulative incidence for discontinuation based on different events: signs of progression, conversion to active treatment without evidence of progression, watchful waiting, death, and still being on AS. An increase in discontinuation can be observed after $1 \mathrm{yr}$, with the largest proportion being due to signs of disease progression and conversion to active treatment without evidence of progression. Moreover, it is worth noting that the proportion of men dying from other causes increased gradually throughout the follow-up, which reflects the real-world setting of this database. Finally, it can be seen that the proportion of men converting to active

Table 2 - Number of patients from each centre in GAP3 at $5 \mathrm{yr}$ of follow-up

\begin{tabular}{|c|c|c|c|c|c|c|c|c|}
\hline Centre & $\begin{array}{l}\text { Still } \\
\text { on AS }\end{array}$ & $\begin{array}{l}\text { Still on AS, } \\
\text { follow-up } \\
\quad<5 \mathrm{yr}\end{array}$ & $\begin{array}{l}\text { Lost to } \\
\text { follow-up }\end{array}$ & Progression & $\begin{array}{l}\text { Converted to } \\
\text { active treatment }\end{array}$ & $\begin{array}{l}\text { Watchful } \\
\text { waiting }\end{array}$ & $\begin{array}{l}\text { Death from } \\
\text { other causes }\end{array}$ & Total \\
\hline EU-Atlanta & 5 & 41 & 0 & 2 & 0 & 0 & 0 & 48 \\
\hline KB-Baden & 44 & 52 & 0 & 22 & 24 & 2 & 1 & 145 \\
\hline UOF-Calgary & 82 & 346 & 0 & 80 & 30 & 0 & 0 & 538 \\
\hline CUHT-Cambridge & 21 & 162 & 14 & 18 & 7 & 1 & 1 & 224 \\
\hline EMC-Rotterdam & 49 & 18 & 3 & 33 & 4 & 5 & 0 & 112 \\
\hline other PRIAS centres & 149 & 1368 & 26 & 392 & 136 & 51 & 13 & 2135 \\
\hline SU-Gothenburg & 293 & 147 & 1 & 111 & 142 & 0 & 43 & 737 \\
\hline HUCH-Helsinki & 58 & 97 & 1 & 97 & 9 & 17 & 3 & 282 \\
\hline JHU-Baltimore & 461 & 315 & 91 & 400 & 141 & 0 & 9 & 1417 \\
\hline KU-Kagawa & 29 & 2 & 1 & 45 & 19 & 3 & 5 & 104 \\
\hline CHU-Lille & 4 & 94 & 10 & 36 & 10 & 0 & 1 & 155 \\
\hline GSTT-London & 58 & 43 & 0 & 83 & 8 & 0 & 2 & 194 \\
\hline UCL-London & 30 & 230 & 0 & 0 & 10 & 2 & 0 & 272 \\
\hline SUS-Malmö & 10 & 90 & 1 & 19 & 4 & 1 & 1 & 126 \\
\hline MEASCAP-Melbourne & 53 & 114 & 0 & 63 & 3 & 3 & 0 & 236 \\
\hline INT-Milan & 102 & 287 & 0 & 245 & 51 & 23 & 2 & 710 \\
\hline MSKCC-New York & 443 & 344 & 0 & 56 & 190 & 0 & 16 & 1049 \\
\hline YUHS-Seoul & 0 & 33 & 0 & 2 & 1 & 0 & 0 & 36 \\
\hline SGH-Singapore & 21 & 93 & 0 & 20 & 46 & 0 & 1 & 181 \\
\hline UCSF-San Francisco & 405 & 487 & 0 & 262 & 94 & 0 & 11 & 1259 \\
\hline IVO-Valencia & 22 & 149 & 0 & 61 & 21 & 10 & 5 & 268 \\
\hline UBC-Vancouver & 0 & 49 & 1 & 14 & 2 & 0 & 2 & 68 \\
\hline Total & 2339 & 4561 & 149 & 2061 & 952 & 118 & 116 & 10296 \\
\hline
\end{tabular}

GAP3 = Movember's Global Action Plan Prostate Cancer Active Surveillance initiative; KCL = King's College London; Erasmus MC=Erasmus Medical Center; MSKCC = Memorial Sloan Kettering Cancer Center; PRIAS = Prostate Cancer Research International Active Surveillance; UCL = University College London; $\mathrm{UCSF}=$ University of California, San Francisco. See appendix A for institute abbreviations. 


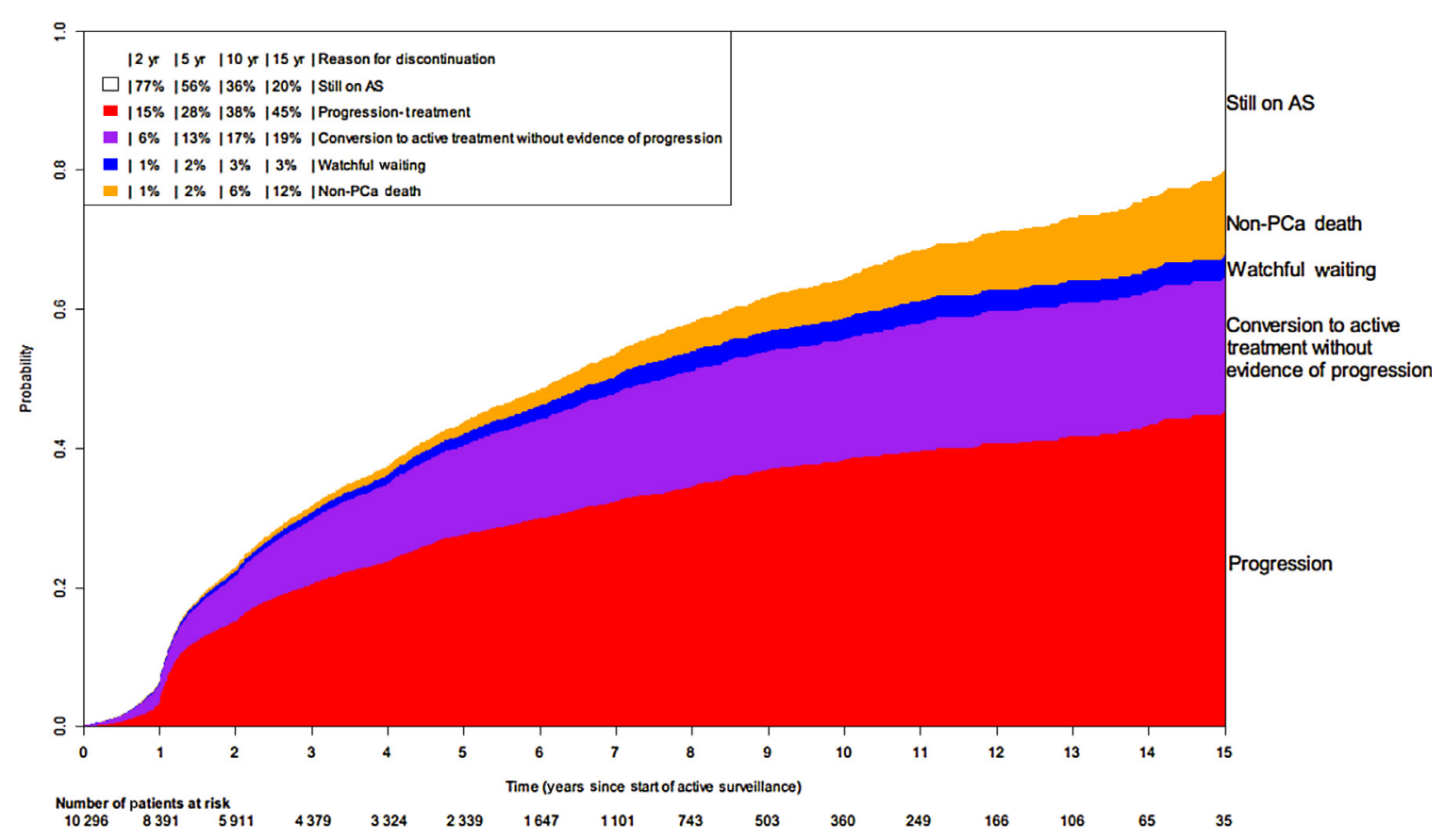

Fig. 1 - Cumulative incidence of discontinuation of active surveillance over time. AS = active surveillance; PCa = prostate cancer.

treatment without evidence of progression remained stable from about 7 yr onwards; a similar trend was observed for conversion to watchful waiting. To further understand how patient characteristics may affect discontinuation of AS, we generated a forest plot specifically focussing on the effects of age (in decade), PSA, and more than one positive biopsy core (Fig. 2). As expected, the strongest positive association is seen for age with transferring to watchful waiting and
non-PCa death. Furthermore, more than one positive biopsy core positively associates with progression and non-PCa death.

Figure 3 shows the cumulative incidence for discontinuation for each centre included in the GAP3 database. For all centres, an increase in signs of disease progression was also observed after $1 \mathrm{yr}$, but the slope of this increase varied substantially by centre.

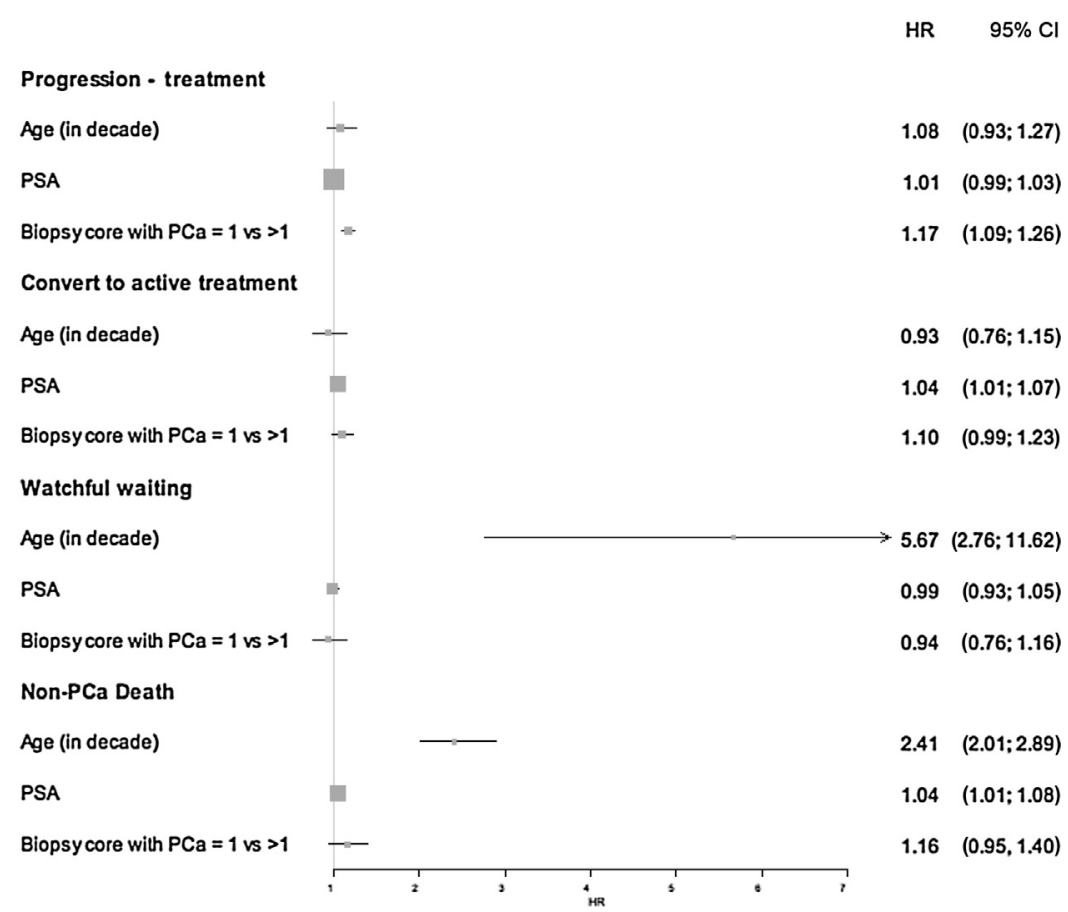

Fig. 2 - Forest plot showing the association between age, PSA, positive biopsy cores, and different reasons for discontinuation of active surveillance. $\mathrm{CI}=$ confidence interval; $\mathrm{HR}=$ hazard ratio; $\mathrm{PCa}=$ prostate cancer; $\mathrm{PSA}=$ prostate-specific antigen. 


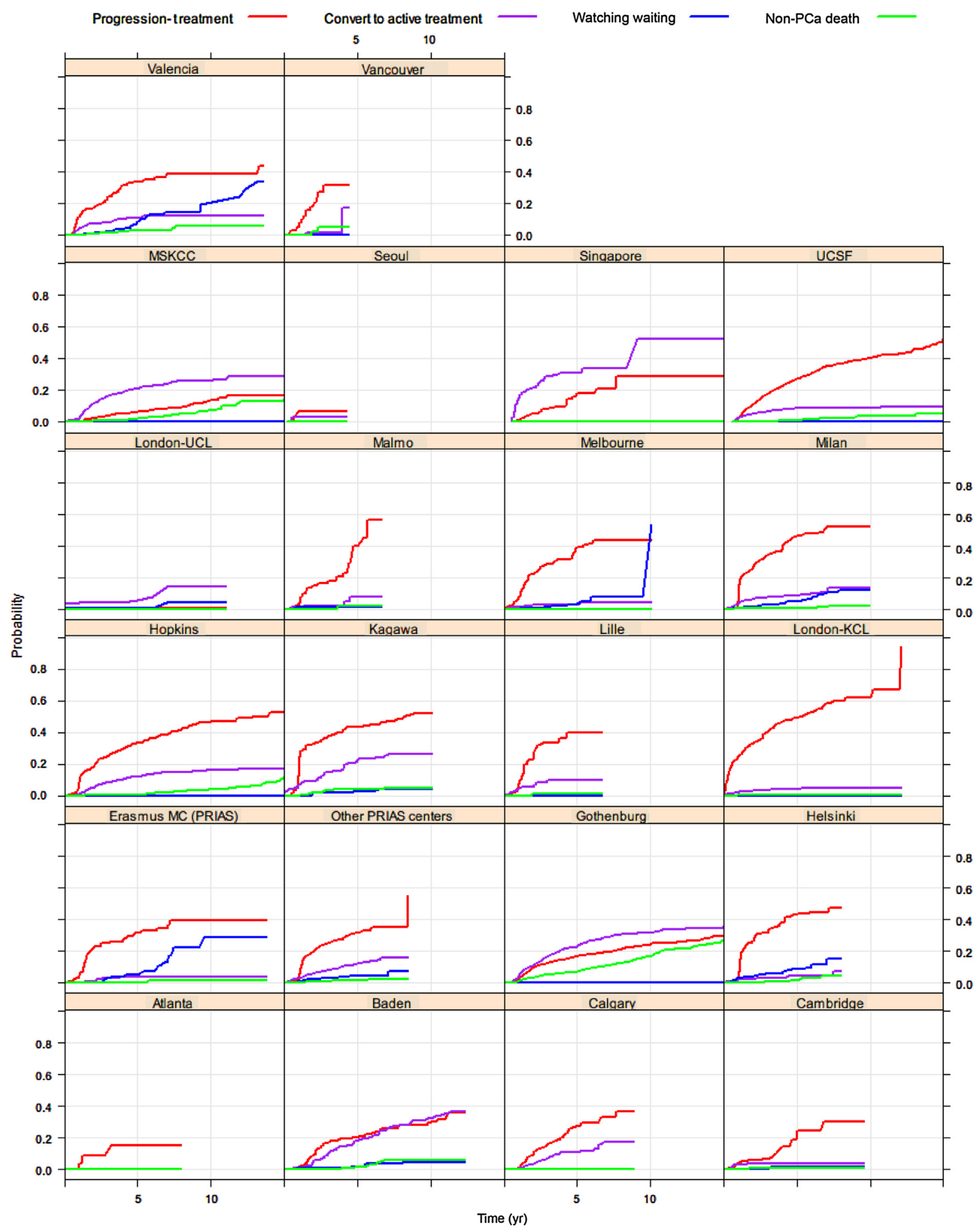

Fig. 3 - Cumulative incidence of discontinuation of active surveillance over time for each centre in the GAP3 database. KCL = King's College London; MC = Erasmus Medical Center; MSKCC = Memorial Sloan Kettering Cancer Center; PCa = prostate cancer; PRIAS = Prostate Cancer Research International Active Surveillance; UCL = University College London; UCSF = University of California, San Francisco.

\section{Discussion}

Based on the data from the largest AS database in the world, we observed that after about $5 \mathrm{yr}$ of follow-up, about $56.4 \%$ of men were still on AS. Substantial variation by centre was observed, but the main reasons for discontinuation were signs of disease progression (27.5\% of men) and conversion to active treatment without evidence of disease progression ( $12.8 \%$ of men). 
As shown in a recent systematic review by Kinsella et al. [12], many factors influence men's adherence to AS on multiple levels. Their thematic assessment of barriers and facilitators for adherence to AS identified many key themes: (1) patient and tumour factors (age, comorbidities, knowledge, education, socioeconomic status, family history, grade, tumour volume, fear of progression/side-effects), (2) family and social support, (3) provider (speciality, communication, attitudes), (4) healthcare organisation (geography, type of practice), and (5) health policy (guidelines, year, awareness) [12]. Interestingly, this systematic review observed that even though a number of studies have shown that emotional distress is relatively high in men at the time of their PC diagnosis [13,14], anxiety in men on long-term AS has been generally reported as favourably low. More studies have suggested that anxiety in men on AS reduces [15-17] or remains the same over time [8,17-22].

Our findings of a drop-out rate of $43.6 \%$ after about $5 \mathrm{yr}$ are in line with previous estimations [23]. However, the proportion of men opting out without evidence for progression was only $12.8 \%$. The variation observed between different institutions shows rather distinct patterns with respect to the proportion of men dropping out due to progression and the proportion of men dropping out due to conversion to active treatment without evidence of disease progression. However, part of the reason why the proportion of drop-out due to conversion to active treatment without evidence of disease progression was largest in Memorial Sloan Kettering Cancer Center, Singapore, Baden, and Goteborg may be explained by the fact that their median follow-up was about $3-4 \mathrm{yr}$ as compared with 1-2 yr in most other centres. Nevertheless, the data from other centres with lengthier follow-up such as John Hopkins University, Valencia, and University of California San Francisco, still showed the largest proportion of discontinuation due to disease progression. In this context, it was also interesting to note that the proportion of men converting to active treatment without evidence of progression in our database remained stable after about $7 \mathrm{yr}$. It can be speculated that more anxious men (and clinicians) were more likely to make the decision about discontinuing AS during the first years. It might suggest that more emphasis on education and support is required during these first years on AS [12]. Surprisingly, the proportion of watchful waiting also stabilised after $7 \mathrm{yr}$, which is unexpected as the population is growing older. Again, this observation might be due to different practices across centres.

The rather large proportion of drop-outs due to signs of disease progression also highlights the need for better inclusion/exclusion criteria, better markers of stable disease, and better outcome measures. For instance, a recent review by Nowinski et al. [24] showed the need for novel approaches of classification, including molecular features, to direct therapy for men with low-grade PCa, especially those on AS. They concluded that by combining genomewide association study data with gene expression and structural rearrangements, risk alleles were identified, which could provide a new basis for developing a prognostication tool to guide therapy for men with early PCa $[24,25]$.

Moreover, the use of MRI as a tool to risk stratify men with low-risk PCa has been emerging over time. A study by Thurtle et al. [26] evaluated data from 157 men enrolled on AS using a protocol including multiparametric MRI, and noted low progression and treatment conversion rates. Changes in multiparametric MRI findings were found to be the principal trigger for detecting progression by imaging alone or pathologically. In addition, the recent findings of the PROMIS trial, which was based on men with PSA concentrations up to $15 \mathrm{ng} / \mathrm{ml}$ and with no previous biopsy, have shown us that MRI identified nearly all men with clinically significant PCa (93\%) versus the current practice standard (transrectal biopsy), which identified only $48 \%$ [27]. The endotype generated by positive MRI was positively associated with grade and volume, and contained cancer in most cases (Likert $\geq 4=92 \%$; Likert $\geq 3=60 \%$ ). An update of the current Movember GAP3 database with information on MRI images will hence provide us more insight into the use of MRI as a selection and monitoring tool for AS.

In addition to genetics and MRI, several studies have also investigated the use of serum biomarkers as a tool to monitor men on AS. However, a recent systematic review by Loeb and Tosoian [28] concluded that very few markers have longitudinal results available yet for men on AS, indicating an important area for future research where the GAP3 database will be able to contribute. Furthermore, simple changes in clinical assessment have been proposed as a strategy to reduce rates of discontinuation of AS. Bokhorst et al. [29] have, for example, shown that the number of positive biopsies should no longer be used to trigger immediate active treatment, but rather to indicate further investigation to confirm the suspicion of higher-risk disease.

The GAP3 database is a unique resource covering data from all over the world. Some limitations exist, resulting in not all centres being included in these analyses due to the lack of information on the reason for discontinuation and limited follow up. However, even after a follow-up of $5 \mathrm{yr}$, we could already observe clear patterns with respect to reasons for discontinuation. The heterogeneity in study protocols and data collection across centres can be seen as a limitation; however, we would like to argue that it is this real-world setting that adds value to our understanding of AS. As outlined by PIONEER, the big PCa data consortium of the European Association of Urology, combination and analysis of the patient records of men diagnosed with PCa can enable healthcare systems to provide more efficient outcome-driven patient-centred interventions [30]. By providing data from a wide variety of centres, GAP3 has the power to transform the perspective of all relevant stakeholders. Recently, Movember has also allocated additional funding to maintain the database and update the clinical data annually, thereby prolonging follow-up time. Furthermore, this provides the opportunity to collect evidence on imaging (MRI), molecular (genomics) markers, patient-related outcomes, and more. In addition, it is worth noting that only qualitative data will not be sufficient to 
answer the question about adherence to AS-there is a need to combine our observations with qualitative studies to truly understand the patterns of discontinuation [12]. Given the available data on the natural course of low-risk disease, the question about whether active monitoring leads to better outcome and benefit whilst avoiding missing the window of cure in case of reclassification/progression is crucial.

\section{Conclusions}

Our descriptive analyses of current AS practices around the world showed that about $43.6 \%$ of men drop out of AS after $5 \mathrm{yr}$, mainly due to signs of disease progression-about $12.8 \%$ of drop-outs were due to conversion to active treatment without evidence of progression. Improvements in selection tools for AS (eg, biomarkers or MRI) are thus needed to correctly allocate men with PCa to AS, which in turn will also reduce discontinuation due to conversion to active treatment without evidence of disease progression.

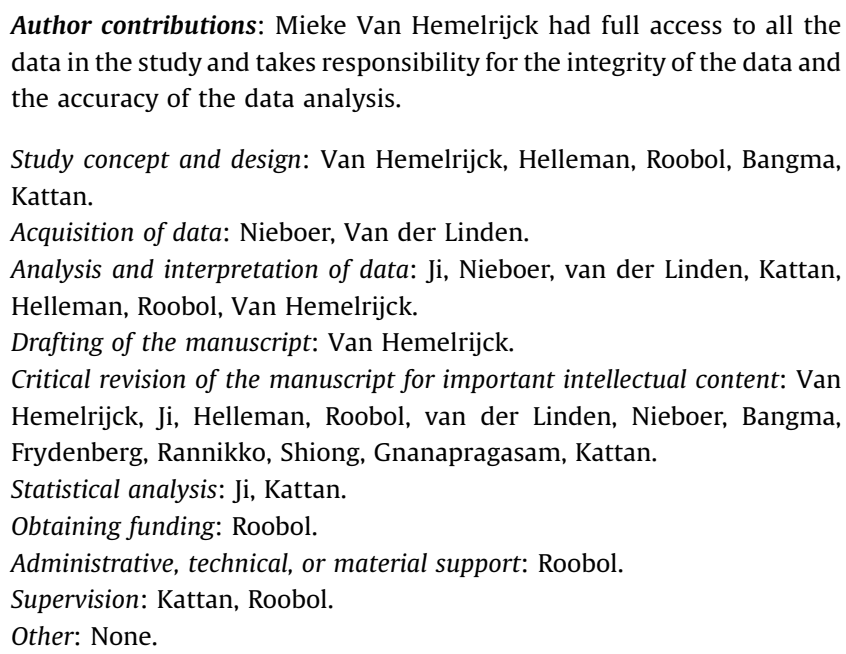

Financial disclosures: Mieke Van Hemelrijck certifies that all conflicts of interest, including specific financial interests and relationships and affiliations relevant to the subject matter or materials discussed in the manuscript (eg, employment/affiliation, grants or funding, consultancies, honoraria, stock ownership or options, expert testimony, royalties, or patents filed, received, or pending), are the following: None.

Funding/Support and role of the sponsor: This work was supported by the Movember Foundation. The funder did not play any role in the study design, collection, analysis, or interpretation of data, or in the drafting of this paper.

\section{Appendix A. Supplementary data}

Supplementary data associated with this article can be found, in the online version, at https://doi.org/10.1016/j. eururo.2018.10.025.

Members of the Movember Foundation's Global Action Plan Prostate Cancer Active Surveillance (GAP3) consortium Principle Investigators: Bruce Trock (Johns Hopkins Univer- sity, The James Buchanan Brady Urological Institute, Baltimore, USA) Behfar Ehdaie (Memorial Sloan Kettering Cancer Center, New York, USA) Peter Carroll (University of California San Francisco, San Francisco, USA): Christopher Filson (Emory University School of Medicine, Winship Cancer Institute, Atlanta, USA) Jeri Kim/Christopher Logothetis (MD Anderson Cancer Centre, Houston, USA)Todd Morgan (University of Michigan and Michigan Urological Surgery Improvement Collaborative (MUSIC), Michigan, USA) Laurence Klotz (University of Toronto, Sunnybrook Health Sciences Centre, Toronto, Ontario, Canada) Tom Pickles (University of British Columbia, BC Cancer Agency, Vancouver, Canada) Eric Hyndman (University of Calgary, Southern Alberta Institute of Urology, Calgary, Canada) Caroline M. Moore (University College London \& University College London Hospital Trust, London, UK) Vincent Gnanapragasam (University of Cambridge \& Cambridge University Hospitals NHS Foundation Trust, Cambridge, UK) Mieke Van Hemelrijck (King's College London, London, UK \& Guy's and St Thomas' NHS Foundation Trust, London, UK) Prokar Dasgupta (Guy's and St Thomas' NHS Foundation Trust, London, UK) Chris Bangma (Erasmus Medical Center, Rotterdam, The Netherlands / Prostate Cancer Research International Active Surveillance (PRIAS) Consortium) Monique Roobol (Erasmus Medical Center, Rotterdam, The Netherlands / Prostate Cancer Research International Active Surveillance (PRIAS) Consortium) Arnauld Villers (Lille University Hospital Center, Lille, France) Antti Rannikko (Helsinki University and Helsinki University Hospital, Helsinki, Finland) Riccardo Valdagni (Department of Oncology and Hemato-oncology, Università degli Studi di Milano, Radiation Oncology 1 and Prostate Cancer Program, Fondazione IRCCS Istituto Nazionale dei Tumori, Milan, Italy) Antoinette Perry (University College Dublin, Dublin, Ireland) Jonas Hugosson (Sahlgrenska University Hospital, Göteborg, Sweden) Jose Rubio-Briones (Instituto Valenciano de Oncología, Valencia, Spain) Anders Bjartell (Skåne University Hospital, Malmö, Sweden) Lukas Hefermehl (Kantonsspital Baden, Baden, Switzerland) Lee Lui Shiong (Singapore General Hospital, Singapore, Singapore) Mark Frydenberg (Monash Health; Monash University, Melbourne, Australia) Yoshiyuki Kakehi (Kagawa University Faculty of Medicine, Kagawa, Japan) Byung Ha Chung (Gangnam Severance Hospital, Yonsei University Health System, Seoul, Republic of Korea). Pathologist:Theo van der Kwast (Princess Margaret Cancer Centre, Toronto, Canada). Technology Research Partners: Henk Obbink (Royal Philips, Eindhoven, The Netherlands) Wim van der Linden (Royal Philips, Eindhoven, The Netherlands) Tim Hulsen (Royal Philips, Eindhoven, The Netherlands) Cees de Jonge (Royal Philips, Eindhoven, The Netherlands). Advisory Regional statisticians: Mike Kattan (Cleveland Clinic, Cleveland, Ohio, USA) Ji Xinge (Cleveland Clinic, Cleveland, Ohio, USA). Kenneth Muir (University of Manchester, Manchester, UK) Artitaya Lophatananon (University of Manchester, Manchester, UK) Michael Fahey (Epworth HealthCare, Melbourne, Australia) Ewout Steyerberg (Erasmus Medical Center, Rotterdam, The Netherlands) Daan Nieboer (Erasmus Medical Center, Rotterdam, The Netherlands) Liying Zhang (University of Toronto, Sunnybrook Health Sciences Centre, Toronto, 
Ontario, Canada). Executive Regional statisticians: Ewout Steyerberg (Erasmus Medical Center, Rotterdam, The Netherlands) Daan Nieboer (Erasmus Medical Center, Rotterdam, The Netherlands) Aida Santa Olalla/Kerri Beckmann (King's College London, London, UK \& Guy's and St Thomas' NHS Foundation Trust, London, UK) Brian Denton (University of Michigan, Michigan, USA) Andrew Hayen (University of Technology Sydney, Australia) Paul Boutros (Ontario Institute of Cancer Research, Toronto, Ontario, Canada). Clinical Research Partners' IT Experts:Wei Guo (Johns Hopkins University, The James Buchanan Brady Urological Institute, Baltimore, USA) Nicole Benfante (Memorial Sloan Kettering Cancer Center, New York, USA) Janet Cowan (University of California San Francisco, San Francisco, USA) Dattatraya Patil (Emory University School of Medicine, Winship Cancer Institute, Atlanta, USA) Emily Tolosa (MD Anderson Cancer Centre, Houston, Texas, USA) Tae-Kyung Kim (University of Michigan and Michigan Urological Surgery Improvement Collaborative, Ann Arbor, Michigan, USA) Alexandre Mamedov (University of Toronto, Sunnybrook Health Sciences Centre, Toronto, Ontario, Canada) Vincent La Pointe (University of British Columbia, BC Cancer Agency, Vancouver, Canada) Trafford Crump (University of Calgary, Southern Alberta Institute of Urology, Calgary, Canada) Jenna Kimberly-Duffell (University of Cambridge \& Cambridge University Hospitals NHS Foundation Trust, Cambridge, UK) Aida Santaolalla (King's College London, London, UK \& Guy's and St Thomas' NHS Foundation Trust, London, UK) Daan Nieboer (Erasmus Medical Center, Rotterdam, The Netherlands) Jonathan Olivier (Lille University Hospital Center, Lille, France) Tiziana Rancati (Fondazione IRCCS Istituto Nazionale dei Tumori di Milano, Milan, Italy) Helén Ahlgren (Sahlgrenska University Hospital, Göteborg, Sweden) Juanma Mascarós (Instituto Valenciano de Oncología, Valencia, Spain) Annica Löfgren (Skåne University Hospital, Malmö, Sweden) Kurt Lehmann (Kantonsspital Baden, Baden, Switzerland) Catherine Han Lin (Monash University and Epworth Health Care, Melbourne, Australia) Hiromi Hirama (Kagawa University, Kagawa, Japan) Kwang Suk Lee (Yonsei University College of Medicine, Gangnam Severance Hospital, Seoul, Korea). Research Advisory Committee:Guido Jenster (Erasmus MC, Rotterdam, The Netherlands) Anssi Auvinen (University of Tampere, Tampere, Finland) Anders Bjartell (Skåne University Hospital, Malmö, Sweden) Masoom Haider (University of Toronto, Toronto, Canada) Kees van Bochove (The Hyve B.V. Utrecht, Utrecht, The Netherlands) Ballentine Carter (Johns Hopkins University, Baltimore, USA). Management team: Sam Gledhill (Movember Foundation, Melbourne, Australia) Mark Buzza (Movember Foundation, Melbourne, Australia) Chris Bangma (Erasmus Medical Center, Rotterdam, The Netherlands) Monique Roobol (Erasmus Medical Center, Rotterdam, The Netherlands) Sophie Bruinsma/Jozien Helleman (Erasmus Medical Center, Rotterdam, The Netherlands).

\section{References}

[1] Albertsen PC. Active surveillance: a ten-year journey. Eur Urol 2017;72:542-3.
[2] Dall'Era MA, Albertsen PC, Bangma C, et al. Active surveillance for prostate cancer: a systematic review of the literature. Eur Urol 2012;62:976-83.

[3] Kinsella N, Helleman J, Bruinsma S, et al. Active surveillance for prostate cancer: a systematic review of contemporary worldwide practices. Transl Androl Urol 2018;7:83-97.

[4] Bokhorst LP, Alberts AR, Rannikko A, et al. Compliance rates with the Prostate Cancer Research International Active Surveillance (PRIAS) protocol and disease reclassification in noncompliers. Eur Urol 2015;68:814-21.

[5] Bruinsma SM, Bangma CH, Carroll PR, et al. Active surveillance for prostate cancer: a narrative review of clinical guidelines. Nat Rev Urol 2016;13:151-67.

[6] Chen RC, Rumble RB, Loblaw DA, et al. Active surveillance for the management of localized prostate cancer (Cancer Care Ontario Guideline): American Society of Clinical Oncology clinical practice guideline endorsement. J Clin Oncol 2016;34:2182-90.

[7] van den Bergh RC, Essink-Bot ML, Roobol MJ, et al. Anxiety and distress during active surveillance for early prostate cancer. Cancer 2009;115:3868-78.

[8] Wilcox C, Gilbourd D, Louie-Johnsun M. Anxiety and health related quality of life (HRQL) in patients undergoing active surveillance of prostate cancer in an Australian centre. BJU Int 2014;113:64-8.

[9] Bergman J, Litwin MS. Quality of life in men undergoing active surveillance for localized prostate cancer. J Natl Cancer Inst Monographs 2012;2012:242-9.

[10] Jiwa M, Halkett G, Meng X, Pillai V, Berg M, Shaw T. Supporting patients treated for prostate cancer: a video vignette study with an email-based educational program in general practice. J Med Internet Res 2014;16:e63.

[11] Bruinsma SM, Zhang L, Roobol MJ, et al. The Movember Foundation's GAP3 cohort: a profile of the largest global prostate cancer active surveillance database to date. BJU Int 2018;121:737-44.

[12] Kinsella N, Stattin P, Cahill D, et al. Factors influencing men's choice of and adherence to active surveillance for low-risk prostate cancer: a mixed-methods systematic review. Eur Urol Suppl 2018;74:261-80.

[13] Korfage I, Essink-Bot M-L, Janssens A, Schröder F, De Koning H. Anxiety and depression after prostate cancer diagnosis and treatment: 5-year follow-up. Br J Cancer 2006;94:1093-8.

[14] Orom H, Underwood III W, Biddle C. Emotional distress increases the likelihood of undergoing surgery among men with localized prostate cancer. J Urol 2017;197:350-5.

[15] Vasarainen H, Lokman U, Ruutu M, Taari K, Rannikko A. Prostate cancer active surveillance and health-related quality of life: results of the Finnish arm of the prospective trial. BJU Int 2012;109:1614-9.

[16] Vanagas G, Mickevičiene A, Ulys A. Does quality of life of prostate cancer patients differ by stage and treatment? Scand J Public Health 2013;41:58-64

[17] van den Bergh RC, Essink-Bot ML, Roobol MJ, Schroder FH, Bangma $\mathrm{CH}$, Steyerberg EW. Do anxiety and distress increase during active surveillance for low risk prostate cancer? J Urol 2010;183:1786-91.

[18] Burnet KL, Parker C, Dearnaley D, Brewin CR, Watson M. Does active surveillance for men with localized prostate cancer carry psychological morbidity? BJU Int 2007;100:540-3.

[19] Davison BJ, Goldenberg SL. Patient acceptance of active surveillance as a treatment option for low-risk prostate cancer. BJU Int 2011;108:1787-93.

[20] Bellardita L, Rancati T, Alvisi MF, et al. Predictors of health-related quality of life and adjustment to prostate cancer during active surveillance. Eur Urol 2013;64:30-6.

[21] Punnen S, Cowan JE, Dunn LB, Shumay DM, Carroll PR, Cooperberg MR. A longitudinal study of anxiety, depression and distress as predictors of sexual and urinary quality of life in men with prostate cancer. BJU Int 2013;112:E67-75. 
[22] Anderson J, Burney S, Brooker JE, et al. Anxiety in the management of localised prostate cancer by active surveillance. BJU Int 2014;114 (Suppl 1):55-61.

[23] Dall'Era MA, Cooperberg MR, Chan JM, et al. Active surveillance for early-stage prostate cancer: review of the current literature. Cancer 2008;112:1650-9.

[24] Nowinski S, Santaolalla A, O'Leary B, et al. Systematic identification of functionally relevant risk alleles to stratify aggressive versus indolent prostate cancer. Oncotarget 2018;9:12812-24.

[25] Cozar JM, Robles-Fernandez I, Martinez-Gonzalez LJ, et al. Genetic markers a landscape in prostate cancer. Mutat Res 2018;775:1-10.

[26] Thurtle D, Barrett T, Thankappan-Nair V, et al. Progression and treatment rates using an active surveillance protocol incorporating image-guided baseline biopsies and multiparametric magnetic resonance imaging monitoring for men with favourable-risk prostate cancer. BJU Int 2018;122:59-65.

[27] Ahmed HU, El-Shater Bosaily A, Brown LC, et al. Diagnostic accuracy of multi-parametric MRI and TRUS biopsy in prostate cancer (PROMIS): a paired validating confirmatory study. Lancet 2017;389:815-22.

[28] Loeb S, Tosoian JJ. Biomarkers in active surveillance. Transl Androl Urol 2018;7:155-9.

[29] Bokhorst LP, Valdagni R, Rannikko A, et al. A decade of active surveillance in the PRIAS study: an update and evaluation of the criteria used to recommend a switch to active treatment. Eur Urol 2016;70:954-60.

[30] European Association of Urology. PIONEER: the European network of excellence for big data in prostate cancer. 2018. https:// prostate-pioneer.eu

\section{CME Educational Centre}

\section{\begin{tabular}{|l} 
EUROPEAN \\
UROLOGY \\
- oowerd taser rogether
\end{tabular}}

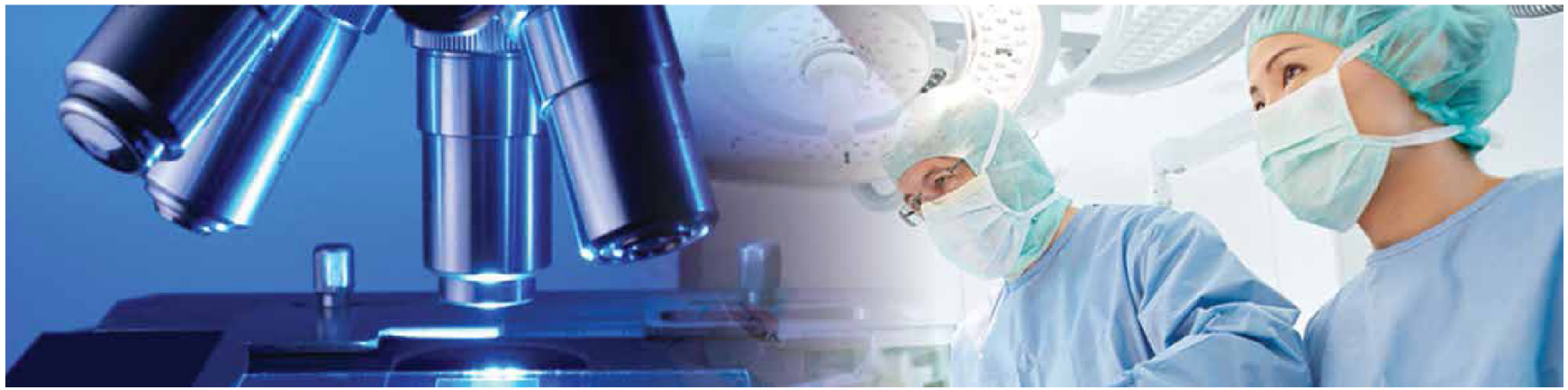

\section{Bladder Cancer Educational Centre}

New interactive CME activities from European Urology. View the accredited activities and take the knowledge tests to obtain CME/CE or simply to assess your skills!

\section{Visit: bladdercancer.cme.europeanurology.com}

